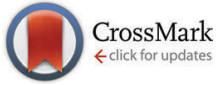

Cite this: Chem. Commun., 2016 52,5573

Received 4th February 2016

Accepted 10th March 2016

DOI: $10.1039 / c 6 c c 01146 b$

www.rsc.org/chemcomm

\section{A silica-based magnetic platform decorated with mixed ligand gold nanoparticles: a recyclable catalyst for esterification reactions $\dagger$}

\author{
Elif Ertem, ${ }^{a}$ Nerea Murillo-Cremaes, ${ }^{b}$ Randy Patrick Carney, ${ }^{a}$ Anna Laromaine, ${ }^{b}$ \\ Emma-Rose Janeček, ${ }^{a}$ Anna Roig ${ }^{\star b}$ and Francesco Stellacci*a
}

\begin{abstract}
A novel and convenient synthetic strategy for the preparation of magnetically responsive silica nanospheres decorated with mixed ligand protected gold nanoparticles is described. Gold nanoparticles are attached to the silica surface via stable amide bond formation. The hierarchical nanospheres show promising results as reusable and efficient catalysts for esterification reactions and they can be recovered through a simple magnetic separation.
\end{abstract}

The combination of different materials in a nanoparticle construct to create novel and multifunctional composite systems has attracted much attention due to their huge potential in various fields of application such as diagnostics and therapy, electronics and catalysis. ${ }^{1-6}$ Recently, our group showed that gold nanoparticles (AuNPs) protected by a monolayer composed of one hydrophobic ligand and one hydrophilic ligand containing terminal sulfonic acid head groups show catalytic potential in esterification reactions. ${ }^{7}$ These mixed ligand coated AuNPs are only mildly dispersible in the reaction medium, so they can be recovered from the solution by centrifugation. However, this is an expensive process that becomes rather cumbersome if all particles from the reaction solution are to be removed.

Here, we present an elegant approach to recover the catalytic gold nanoparticles from the solution with a simple magnet and within a few seconds. Starting from a core@shell structure composed of superparamagnetic $\gamma-\mathrm{Fe}_{2} \mathrm{O}_{3}$ (maghemite) nanoparticles coated with $\mathrm{SiO}_{2}$ and taking advantage of the chemical versatility of the silica materials, ${ }^{8-10}$ we surface-functionalized them with amine to anchor the catalytically active AuNPs. We present the synthesis of these $\mathrm{Fe}_{2} \mathrm{O}_{3} @ \mathrm{SiO}_{2} @ \mathrm{Au}$ hierarchical nanospheres and verify that the AuNPs bonded to the silica retain their catalytic activity. These hierarchical nano-constructs

\footnotetext{
${ }^{a}$ Institute of Materials, Ecole Polytechnique Fédérale de Lausanne, Lausanne CH-1015, Switzerland. E-mail: francesco.stellacci@epfl.ch

${ }^{b}$ Institut de Ciència de Materials de Barcelona (ICMAB-CSIC), Campus UAB, Bellaterra, Catalonia, E-08193 Spain.E-mail: roig@icmab.es

$\dagger$ Electronic supplementary information (ESI) available: Experimental details and supplementary figures. See DOI: 10.1039/c6cc01146b
}

lead to some benefits when compared with their gold counterparts: while the silica layer confers water dispersibility to the nanospheres, the magnetic core allows us to attract and remove them from the reaction medium by a magnet facilitating the reusability of the material. This multifunctional composite emerges as an ideal combination of silica, iron oxide and gold to obtain a recyclable and efficient catalyst for the esterification of carboxylic acids.

In this study, AuNPs coated with mixed 3-mercaptopropane1-sulfonic acid (MPSA) and octanethiol (OT) ligands are used as this combination was proven to be the most effective catalyst for esterification reactions. ${ }^{7,11}$ MPSA:OT covered AuNPs are synthesized using a modified version of the one-phase Brust-

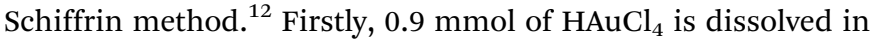
$150 \mathrm{~mL}$ of ethanol in a clean round-bottom flask. In a separate vial, $0.50 \mathrm{mmol}$ of 3-mercapto-1-propanesulfonic acid sodium salt (MPSA) and $0.25 \mathrm{mmol}$ of 1-octanethiol (OT) are mixed in $10 \mathrm{~mL}$ of methanol and added to the stirring gold solution. After 10 min of stirring at room temperature, $150 \mathrm{~mL}$ of $\mathrm{NaBH}_{4}$ (378.1 $\mathrm{mg}$ ) is added dropwise to the reaction flask. The reaction is stirred for $2 \mathrm{~h}$ more before placing it at $4{ }^{\circ} \mathrm{C}$ overnight in order to precipitate the particles. The supernatant is removed, and the particles are cleaned of impurities through sequential centrifugation in acetone, ethanol and methanol. Then, the pellet is dried overnight under vacuum.

Thermogravimetric Analysis (TGA) is used to quantify the percentage of organic material on the MPSA:OT covered AuNPs. (Fig. 1). The mass loss below $200{ }^{\circ} \mathrm{C}$ is due to solvent evaporation and corresponds to a weight loss below $1.0 \%$. The two main stages of weight loss in the temperature ranges of $200-400{ }^{\circ} \mathrm{C}$ and $400-650{ }^{\circ} \mathrm{C}$ can be attributed to the decomposition of OT (7.5\%) and MPSA (4.7\%), respectively. Thus, the estimated mass percentage of the total ligand shell is $\sim 12 \%$. A TEM image of MPSA:OT protected AuNPs is included in the inset of Fig. 1 showing their spherical shape and a mean size of $3 \pm 0.8 \mathrm{~nm}$. The MPSA: OT ligand molar ratio on the surface of AuNPs is determined by ${ }^{1} \mathrm{H}$ nuclear magnetic resonance $\left({ }^{1} \mathrm{H} \mathrm{NMR}\right)$ after etching the gold core with iodine solution (ESI, $\dagger$ Fig. S1). The peak at $2.20 \mathrm{ppm}$ 


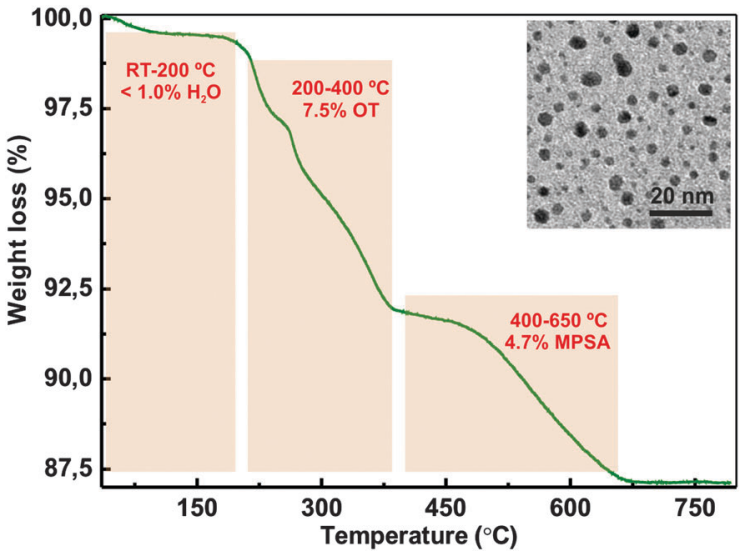

Fig. 1 TGA curve of MPSA:OT covered AuNPs. The inset shows a TEM image of MPSA:OT covered AuNPs.

corresponds to $\beta-\mathrm{CH}_{2}$ neighbouring to the sulfonate on the 3-mercapto-1-propanesulfonate (MPSA) ligand, whereas the $-\mathrm{CH}_{3}$ peak of the 1-octanethiol (OT) ligand appears at $0.92 \mathrm{ppm}$. The ${ }^{1} \mathrm{H}$ NMR spectra of the particles before the etching process show that those particles are free of impurities (data not shown). Based on the calculations using their peak ratio, the ratio of MPSA: OT on the surface of the particles is determined. Although the nominal molar ratio of MPSA: OT ligands is $2: 1$, the experimentally obtained result showing the global ligand composition of the sample is $8: 1$. From the results acquired by TGA, TEM and ${ }^{1} \mathrm{H}$ NMR, the ligand density of MPSA:OT protected AuNPs is found to be 4.8 ligand molecules per $\mathrm{nm}^{2}$.

Once the MPSA:OT protected AuNPs are synthesized, a partial ligand exchange reaction is performed between the MPSA/OT ligands and $N$-hydroxysuccinimidyl 11-mercaptoundecanoate (ProChimia Surfaces) using a modified version of a method previously reported by us. ${ }^{13}$ The concentration of $N$-hydroxysuccinimidyl 11-mercaptoundecanoate is adjusted in such a way that it displaces a few ligands from the surface. ${ }^{14} 10 \mathrm{mg}$ of MPSA:OT protected AuNPs is dissolved in $2.0 \mathrm{~mL}$ of MQ water. In a separate vial, $5 \mathrm{mg}$ of $\mathrm{N}$-hydroxysuccinimidyl 11-mercaptoundecanoate dissolved in $0.5 \mathrm{~mL}$ of dimethylformamide and added to the AuNP solution. After 30 min of stirring, particles are cleaned with a SephadexTM LH-20 column (GE Healthcare) and washed with water. Fig. 2a shows the schematic representation for the partial ligand functionalization of MPSA:OT covered AuNPs.

To prepare the core@shell nanospheres, the superparamagnetic maghemite $\left(\gamma-\mathrm{Fe}_{2} \mathrm{O}_{3}\right)$ nanoparticles composing the cores are first synthesized by the thermal decomposition of iron acetylacetonate $\left(\mathrm{Fe}(\mathrm{acac})_{3}\right)$ in benzyl ether using oleic acid and oleylamine as capping ligands. ${ }^{14,15}$ The as-obtained nanoparticles are uniform and spherical in shape with a mean diameter of $5 \pm 1 \mathrm{~nm}$ (ESI, $\dagger$ Fig. S2a). The magnetic nanoparticles are then encapsulated by a supercritical assisted sol-gel reaction previously reported by us. ${ }^{16,17}$ Tetramethylorthosilicate (TMOS) is used as a silicon precursor, no catalyst is needed for the formation of silica. The process is schematized in Fig. 2b. The resulting core@shell nanospheres (180 $\pm 20 \mathrm{~nm}$ in diameter) are displayed in Fig. S2b (ESI $\dagger$ ). The mass portion of $\mathrm{Fe}_{2} \mathrm{O}_{3}$ with respect to the total mass is a)
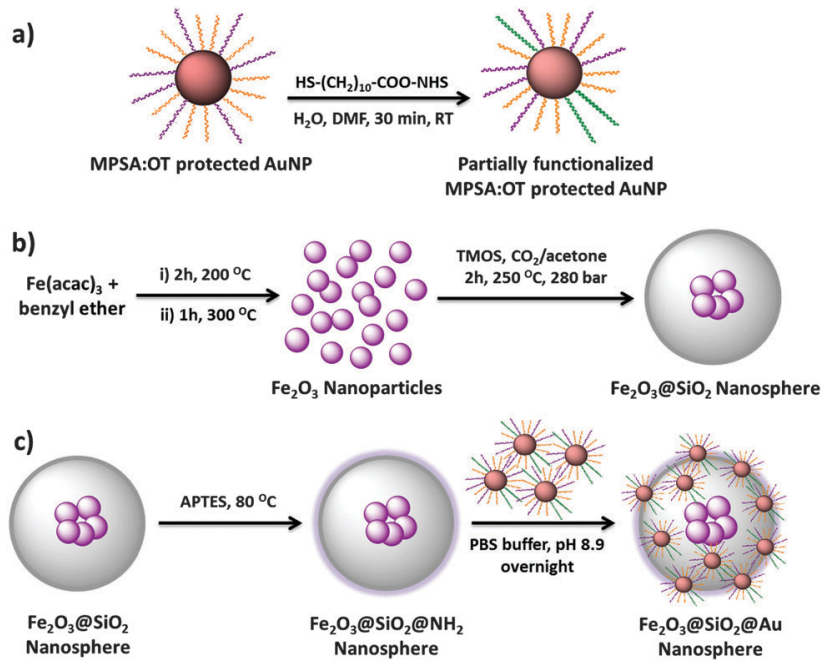

Fig. 2 Schematic illustration of: (a) the partial ligand exchange of MPSA:OT covered AuNPs, (b) the synthesis of $\mathrm{Fe}_{2} \mathrm{O}_{3} \mathrm{CSiO}_{2}$ and (c) the synthesis of $\mathrm{Fe}_{2} \mathrm{O}_{3} @ \mathrm{aSiO}_{2} @ \mathrm{Au}$ hierarchical nanospheres.

9.2\% by ICP-MS. ${ }^{18}$ The magnetic properties of the core@shell material are studied. The saturation magnetization value at $300 \mathrm{~K}$ is $5.4 \mathrm{emu} \mathrm{g}^{-1}$ of $\mathrm{Fe}_{2} \mathrm{O}_{3} @ \mathrm{SiO}_{2}$ (ESI, $\dagger$ Fig. S3a). This value converts to $59 \mathrm{emu} \mathrm{g}^{-1}$ when referred to the mass of $\mathrm{Fe}_{2} \mathrm{O}_{3}$ and is comparable to other reported data. ${ }^{19}$ The absence of coercivity and remanence at room temperature indicates the superparamagnetic character of the composite nanospheres, which behave as ferrimagnets at $5 \mathrm{~K}$, as observed from the hysteresis loop in the inset of Fig. S3a (ESI $\dagger$ ). The zero-field-cooled and field-cooled magnetization curves (ZFC-FC; ESI, $\dagger$ Fig. S3b) highlight the narrow size distribution of the magnetic clusters and confirms the superparamagnetism of the nanospheres with blocking temperature $\left(T_{\mathrm{B}}\right)=61 \mathrm{~K}$. The strong magnetic response of the material enables its easy recovery from the reaction medium using an external magnet.

The $\mathrm{Fe}_{2} \mathrm{O}_{3} @ \mathrm{SiO}_{2}$ nanospheres are then functionalized with 3-(aminopropyl)triethoxysilane (APTES), and a modified version of a synthesis previously reported by us is used. ${ }^{7} 200 \mathrm{mg}$ of $\mathrm{Fe}_{2} \mathrm{O}_{3} @ \mathrm{SiO}_{2}$ nanospheres is dispersed in $350 \mathrm{~mL}$ of ethanol for $20 \mathrm{~min}$ via ultrasonication, and then $2 \mathrm{~mL}$ of APTES is added to the reaction mixture. The reaction is allowed to proceed at $80{ }^{\circ} \mathrm{C}$ for $5 \mathrm{~h}$. The amino modified $\mathrm{Fe}_{2} \mathrm{O}_{3} @ \mathrm{SiO}_{2}$ nanospheres are washed three times with ethanol, and then dried under vacuum for $24 \mathrm{~h}$.

Finally, the formation of the $\mathrm{Fe}_{2} \mathrm{O}_{3} @ \mathrm{SiO}_{2} @ \mathrm{Au}$ nanospheres is conducted. The $N$-hydroxysuccinimide ester functional group allows MPSA:OT protected AuNPs to react with $-\mathrm{NH}_{2}$ groups on the surface of $\mathrm{Fe}_{2} \mathrm{O}_{3} @ \mathrm{SiO}_{2}$ nanospheres to form stable amide bonds in basic aqueous media. Firstly, $50 \mathrm{mg}$ of $\mathrm{Fe}_{2} \mathrm{O}_{3} @ \mathrm{SiO}_{2} @ \mathrm{NH}_{2}$ spheres are dissolved in $38.5 \mathrm{~mL}$ of water and then $5 \mathrm{~mL}$ of $10 \times$ PBS buffer ( $\mathrm{pH}$ 8.9, Ambion) is added to the reaction medium. After $10 \mathrm{~min}$ of sonication, $8.5 \mathrm{mg}$ of MPSA:OT coated AuNPs in $6.5 \mathrm{~mL}$ of water is added and stirred overnight at room temperature in the dark. The resulting hierarchical nanospheres are then cleaned five times with water via magnetic decantation. The synthesis of 
the $\mathrm{Fe}_{2} \mathrm{O}_{3} @ \mathrm{SiO}_{2} @ \mathrm{Au}$ spheres (hereafter hierarchical nanospheres) is schematically presented in Fig. 2c.

The as-prepared hierarchical nanospheres are visualized using TEM, as shown in Fig. 3. Clearly, the silica surface is uniformly covered with MPSA:OT protected AuNPs. It should be noted that AuNPs are successfully attached to the $\mathrm{Fe}_{2} \mathrm{O}_{3} @ \mathrm{SiO}_{2}$ nanospheres only when functionalized with $N$-hydroxysuccinimidyl 11-mercaptoundecanoate; a control experiment led to silica nanospheres almost free of AuNPs (ESI, $\dagger$ Fig. S4). The efficiency of the coupling reaction is remarkable given that the $N$-hydroxysuccinimidyl 11-mercaptoundecanoate ligand is only added to the MPSA:OT covered AuNPs only in a low fraction, indeed its signal could not be detected in ${ }^{1} \mathrm{H}$ NMR even after decomposition of the gold core.

To test the catalytic efficiency of hierarchical nanosphere acetylation of benzyl alcohol $(\mathrm{BzOH})$ is chosen as a test reaction $(\mathrm{ESI} \dagger)$ since the product benzyl acetate (BzAc) is a widely used compound in the industry as a solvent, odorant, plasticizer, and also as a precursor for many agrochemicals, pharmaceuticals, and further fine chemicals. ${ }^{1}$ Prior to the reaction, the terminal sulfonate head groups of the ligand on the monolayer of AuNPs are acidified. The acidification process of sulfonate groups is adapted from the literature (ESI $\dagger)^{1,2}$ Without this step, no catalytic efficiency is observed (data not shown). Sulphuric acid does not cleave the amide bonds ${ }^{20}$ therefore the attachment of MPSA:OT covered AuNPs to the silica surface is stable under the acidification process.

The catalytic reaction is conducted in a $10 \mathrm{~mL}$ round-bottom flask fitted with a reflux condenser. Efficiencies are evaluated after the reaction of $270 \mathrm{mg}(2.5 \mathrm{mmol})$ of $\mathrm{BzOH}$ (Sigma Aldrich, anhydrous), $300 \mathrm{mg}(2.5 \mathrm{mmol})$ of acetic acid (AcOH) (Sigma Aldrich, 99.99\% purity) and $40 \mathrm{mg}$ of the hierarchical nanospheres at $100{ }^{\circ} \mathrm{C}$ for $3 \mathrm{~h}$. To investigate the activities of MPSA:OT covered AuNPs and $\mathrm{Fe}_{2} \mathrm{O}_{3} @ \mathrm{SiO}_{2}$ nanospheres, $5 \mathrm{mg}$ and $40 \mathrm{mg}$ of particles, respectively, are used under the same conditions. After separation of the particles from the reaction medium $\left(\mathrm{Fe}_{2} \mathrm{O}_{3} @ \mathrm{SiO}_{2}\right.$ and hierarchical nanospheres are recovered

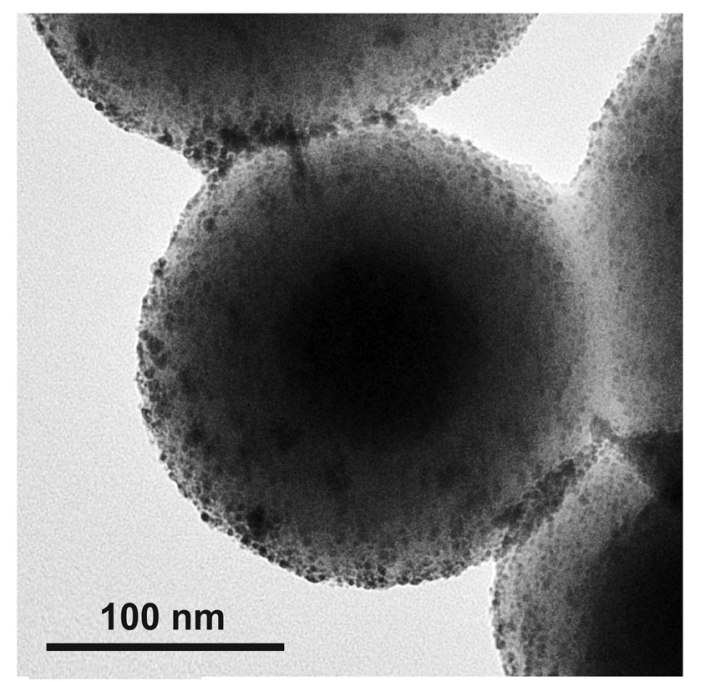

Fig. 3 TEM images of hierarchical $\mathrm{Fe}_{2} \mathrm{O}_{3} \mathrm{CSiO}_{2} @ \mathrm{~A} A u$ nanospheres. via external magnet while MPSA:OT covered AuNPs are separated by centrifugation) the products are analysed by means of ${ }^{1} \mathrm{H}$ NMR. The products are kept at $-4{ }^{\circ} \mathrm{C}$ prior to analyze them simultaneously. The recovered hierarchical nanospheres are then washed with water, and poured in the acidification reaction medium again to be used in a subsequent reaction under similar conditions. This process is performed twice.

Conversion ratios of $\mathrm{BzOH}$ to the product BzAc are calculated as percentages in order to evaluate the catalytic efficacy of hierarchical nanospheres. The results are presented in Fig. 4. The control experiment, which is performed without the addition of any nanoparticles, gives less than $3 \%$ conversion of $\mathrm{BzOH}$ to BzAc (ESI, $\dagger$ Fig. S5). $\mathrm{Fe}_{2} \mathrm{O}_{3} @ \mathrm{SiO}_{2}$ nanospheres show also similar results to the control experiment in terms of conversion efficiency (5\%) as expected since Brønsted acidity of surface hydroxyl groups of the nanospheres is not strong enough to act as a source for the protons to be transferred in an acid catalysis system (ESI, † Fig. S6).

The hierarchical nanospheres (46\%) show a much higher efficiency than MPSA:OT covered AuNPs (28\%) as it can be observed in Fig. 4 and in ESI $\dagger$ (Fig. S7 and Fig. S8). Conversion efficiencies were achieved by using the same quantity of MPSA:OT covered AuNPs in the reaction medium, as determined by optical absorption (ESI $\dagger$ ). Two reasons may explain the superior activity of the hierarchical nanospheres. Firstly, MPSA:OT coated AuNPs are not colloidally dispersible in the reaction medium, whilst the hierarchical nanospheres are very well dispersed and act as both homogeneous and heterogeneous catalysts (ESI, $\dagger$ Fig. S11). Since the hierarchical nanospheres are homogeneously dispersed

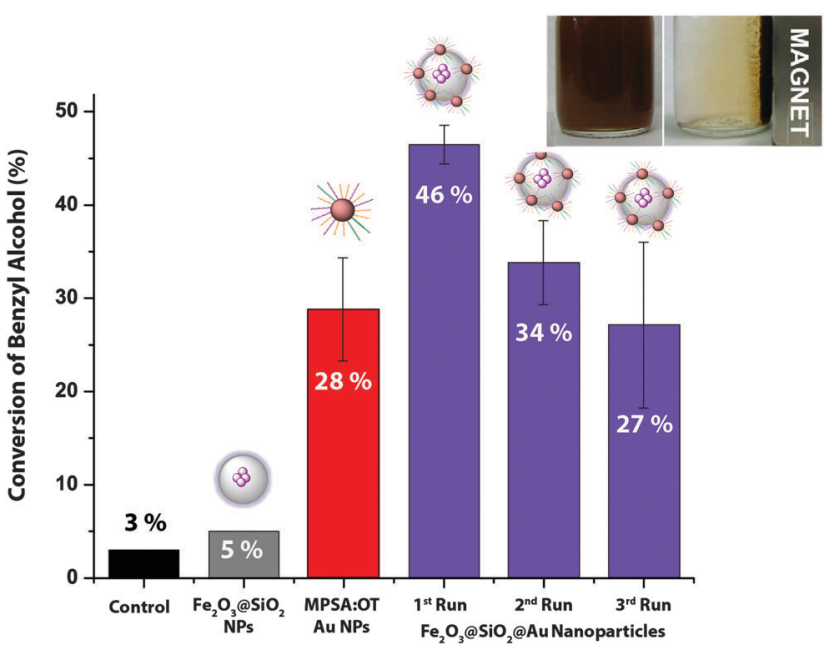

Fig. 4 Efficiency and reusability of MPSA:OT protected AuNPs, $\mathrm{Fe}_{2} \mathrm{O}_{3} \mathrm{C}$ $\mathrm{SiO}_{2}$ nanospheres and hierarchical $\mathrm{Fe}_{2} \mathrm{O}_{3} @ \mathrm{SiO}_{2} @ \mathrm{Au}$ nanospheres as catalysts for the acetylation reaction of benzyl alcohol. The inset displays the photographs of the aqueous dispersion of $\mathrm{Fe}_{2} \mathrm{O}_{3} \mathrm{CSiO}_{2}$ nanospheres without and with an $0.5 \mathrm{~T}$ magnet. The conversion efficiencies of the hierarchical nanospheres and MPSA:OT covered AuNPs are averages of three independent measurements (i.e. three batches independently synthesized). Conversions were calculated starting from the same amount of $\mathrm{Au}$ NPs, the increase in efficiency from MPSA:OT Au NPs to the 1st run of $\mathrm{Fe}_{2} \mathrm{O}_{3} @ \mathrm{SiO}_{2} \mathrm{QAu}$ spheres is most probably due to the fact that the MPSA:OT Au NPs are not colloidally stable in the reaction mixture while the $\mathrm{Fe}_{2} \mathrm{O}_{3} @ \mathrm{CSiO}_{2} @ \mathrm{Au}$ spheres are (ESI, $\dagger$ Fig. S11). 
in the reaction medium, MPSA ligands located at the periphery of the self-assembled monolayer (SAM) behave as if molecularly dissolved. Secondly, since MPSA:OT covered AuNPs are attached to the silica surface forming a homogeneously distributed uniform layer (without creating aggregates), interactions of the sulfonate head groups with the substrate were promoted in higher amounts in the case of hierarchical nanospheres when compared with MPSA:OT protected AuNPs.

In order to examine the reusability of the synthesized hierarchical nanospheres, the particles undergo two more cycles. Fig. 4 shows that hierarchical nanospheres maintain their ability to catalyse the reaction with high efficiency compared to the control experiment after several uses $(34 \%$ for the second run and $27 \%$ for the third run), as shown in Fig. S9 and Fig. S10 (ESI $\dagger$ ). However, there is a decrease in the activity after each run. The third run exhibits a similar catalytic activity compared to MPSA:OT covered AuNPs. To understand the reason behind this decrease, a TEM sample of hierarchical nanospheres is prepared from the third acidification reaction prior to the cleaning step (magnetic decantation). Free AuNPs identified on the TEM images suggest that this loss can be attributed to the decrease in the number of MPSA:OT covered AuNPs on the surface of hierarchical nanospheres after each run. On the other hand, there is no pronounced morphological change of hierarchical nanospheres (ESI, $\dagger$ Fig. S12). We consider that the detachment of the MPSA:OT covered AuNPs from the silica surface might be caused by the desorption of the thiol group of the anchoring ligand, $N$-hydroxysuccinimidyl 11-mercaptoundecanoate, from the gold surface.

In summary, we present a novel approach for synthesizing well-defined $\mathrm{Fe}_{2} \mathrm{O}_{3} @ \mathrm{SiO}_{2} @ \mathrm{Au}$ nanospheres. These hierarchical nanospheres are promising catalytic agents for esterification reactions since they can be recovered and reused with the help of an external magnet and they display characteristics of both homogenous and heterogeneous catalysts. They showed a pronounced increase on the conversion of $\mathrm{BzOH}$ to BzAc compared to the MPSA:OT covered AuNPs. Studies to improve the catalytic efficiency of these nanospheres further are in progress.
This research was partially funded by Spanish Ministry of Economy (MAT2012-35324, Ramon y Cajal grant (AL) RYC-201006082), People Program of the European Commission (grant agreement no. 303630, co-funded by the European Social Fund) and the Generalitat de Catalunya (2014SGR213). Support from the Swiss National Science Foundation is gratefully acknowledged.

\section{Notes and references}

1 F. Caruso, Adv. Mater., 2001, 13, 11.

2 E. Ertem, A. Bekdemir, A. Atilgan and E. U. Akkaya, Pure Appl. Chem., 2014, 86, 899.

3 X. Xia, J. Tu, Y. Zhang, X. Wang, C. Gu, X.-B. Zhao and H. J. Fan, ACS Nano, 2012, 6, 5531.

4 W. Hu, B. Liu, Q. Wang, Y. Liu, Y. Liu, P. Jing, S. Yu, L. Liu and J. Zhang, Chem. Commun., 2013, 49, 7596.

5 B. Liu, D. Zhang, J. Wang, C. Chen, X. Yang and C. Li, J. Phys. Chem. $C, 2013,117,6363$.

6 H. Naeimi and S. Mohamadabadi, Dalton Trans., 2014, 43, 12967.

7 A. Ghosh, S. Basak, B. H. Wunsch, R. Kumar and F. Stellacci, Angew. Chem., Int. Ed. Engl., 2011, 50, 7900.

8 S. Williams, A. Neumann, I. Bremer, Y. Su, G. Dräger, C. Kasper and P. Behrens, J. Mater. Sci.: Mater. Med., 2015, 26, 125.

9 G. Zhang, L. Qin, Y. Wu, Z. Xu and X. Guo, Nanoscale, 2015, 7, 1102.

10 N. Murillo-Cremaes, P. López-Aranguren, L. F. Vega, J. Saurina, A. Roig, C. Domingo and J. Polym. Sci. A, Polym. Chem., 2014, $52,2760$.

11 A. Ghosh, F. Stellacci and R. Kumar, Catal. Today, 2012, 198, 77.

12 M. Brust, J. Fink, D. Bethell, D. J. Schiffrin and C. Kiely, J. Chem. Soc., Chem. Commun., 1995, 1655.

13 G. A. DeVries, F. R. Talley, R. P. Carney and F. Stellacci, Adv. Mater., 2008, 20, 4243.

14 S. Sun, H. Zeng, D. B. Robinson, S. Raoux, P. M. Rice, S. X. Wang and G. Li, J. Am. Chem. Soc., 2004, 126, 273.

15 E. Taboada, E. Rodríguez, A. Roig, J. Oró, A. Roch and R. N. Muller, Langmuir, 2007, 23, 4583.

16 E. Taboada, R. Solanas, E. Rodríguez, R. Weissleder and A. Roig, Adv. Funct. Mater., 2009, 19, 2319.

17 N. Murillo-Cremaes, A. M. López-Periago, J. Saurina, A. Roig and C. Domingo, J. Supercrit. Fluids, 2013, 73, 34.

18 N. Murillo-Cremaes, Multifunctional silica-based nanoparticles for biomedical applications, Doctoral thesis, Universitat Autònoma de Barcelona (UAB), 2014.

19 S. Layek, A. Pandey, A. Pandey and H. C. Verma, Int. Trans. J. Eng., Manage., Appl. Sci. Technol., 2010, 2, 33.

20 G. Fraenel and C. Franconi, J. Am. Chem. Soc., 1960, 82, 4478. 